\title{
Personalized analysis of breast cancer using sample-specific networks
}

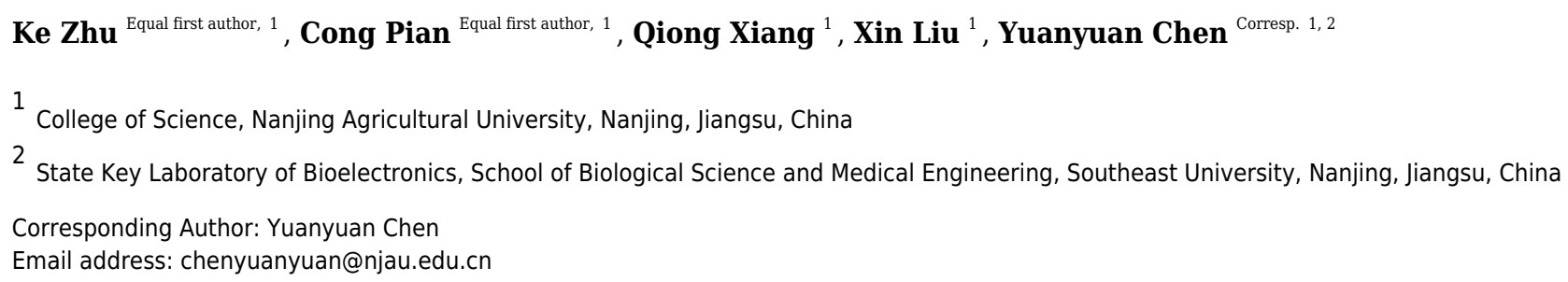

Breast cancer is a disease with high heterogeneity. Cancer is not usually caused by a single gene, but by multiple genes and their interactions with others and surroundings. Estimating breast cancer-specific gene-gene interaction networks is critical to elucidate the mechanisms of breast cancer from a biological network perspective. In this study, sample-specific gene-gene interaction networks of breast cancer samples were established by using a sample-specific network analysis method based on gene expression profiles. Then, gene-gene interaction networks and pathways related to breast cancer and its subtypes and stages were further identified. The similarity and difference among these subtype-related (and stage-related) networks and pathways were studied, which showed highly specific for subtype Basal-like and Stage IV and V. Finally, gene pairwise interactions associated with breast cancer prognosis were identified by a Cox proportional hazards regression model, and a risk prediction model based on the gene pairs was established, which also performed very well on an independent validation data set. This work will help us to better understand the mechanism underlying the occurrence of breast cancer from the sample-specific network perspective. 


\section{Personalized analysis of breast cancer using sample- 2 specific networks}

$5 \mathrm{Ke} \mathrm{Zhu}^{1}$, Cong Pian ${ }^{1}$, Qiong Xiang ${ }^{1}$, Xin Liu ${ }^{1}$, Yuanyuan Chen ${ }^{1,2}$

$7{ }^{1}$ College of Science, Nanjing Agricultural University, Nanjing, Jiangsu, China

$8{ }^{2}$ State Key Laboratory of Bioelectronics, School of Biological Science and Medical

9 Engineering, Southeast University, Nanjing, Jiangsu, China

10

Corresponding Author:

Yuanyuan Chen ${ }^{1,2}$

Xuanwu District Xiaolingwei Street Weigang No.1, Nanjing, Jiangsu, 210095, China

Email address: chenyuanyuan@njau.edu.cn

\section{Abstract}

Breast cancer is a disease with high heterogeneity. Cancer is not usually caused by a single gene, but by multiple genes and their interactions with others and surroundings. Estimating breast cancer-specific gene-gene interaction networks is critical to elucidate the mechanisms of breast cancer from a biological network perspective. In this study, sample-specific gene-gene interaction networks of breast cancer samples were established by using a sample-specific network analysis method based on gene expression profiles. Then, gene-gene interaction networks and pathways related to breast cancer and its subtypes and stages were further identified. The similarity and difference among these subtype-related (and stage-related) networks and pathways were studied, which showed highly specific for subtype Basal-like and Stage IV and V. Finally, gene pairwise interactions associated with breast cancer prognosis were identified by a Cox proportional hazards regression model, and a risk prediction model based on the gene pairs was established, which also performed very well on an independent validation data set. This work will help us to better understand the mechanism underlying the occurrence of breast cancer from the sample-specific network perspective. 


\section{Introduction}

33

34

35

36

37

38

39

40

41

42

43

44

45

46

47

48

49

50

51

52

53

54

55

56

57

58

59

60

61

62

63

64

65

66

67

68

According to the latest data from the survey of the International Agency for Research on Cancer (IARC) in 2018, the incidence of breast cancer is $24.2 \%$ among women worldwide, ranking first in female cancers [1]. At present, the incidence of breast cancer is the highest, and its mortality ranks fourth in China. Breast cancer has strong heterogeneity. Based on the TNM staging system, breast cancer can be divided into Stages I, II, III, IV, and V. There are many clinical types of breast cancer according to pathological classification and molecular classification. The pathological classification generally divides breast cancer into invasive and non-invasive breast cancer. And the gold standard for the molecular typing of breast cancer is PAM50 molecular typing based on the expression profile of 50 genes, which classifies breast cancer into the Normal-like, LuminalA, LuminalB, Basal-like, and Her2 subtypes [2].

The molecular typing of breast cancer has important reference value for clinical treatment of breast cancer. However, molecular typing requires transcription sequencing which is difficult to promote clinically. Currently, the diagnosis of breast cancer classification is mainly through immunohistochemistry (IHC), namely, diagnosis by the expression of four markers, ER (oestrogen receptor), PR (progestin receptor), HER2 gene (human epidermal growth factor receptor 2) and Ki-67 protein (proliferating cell nuclear antigen). ER and PR are important indicators for endocrine therapy and prognosis evaluation in breast cancer. Studies have shown that their expression are positively correlated with total survival, treatment failure time, endocrine therapy response time, and recurrence time [3, 4]. In 2009, Cheang used GEP (gene expression analysis) to determine $14 \%$ as the threshold of Ki-67, which could be used to divide patients into two groups with good and bad prognoses [5]. In 2011, the St. Gallen International Expert Consensus agreed to include $\mathrm{Ki}-67$ as an important standard for molecular typing, which is the key to distinguishing the Luminal A and Luminal B subtypes [6]. In the growth and metastasis of breast cancer, HER2 is one of the most important factors, and its status can be used to predict the effect of drug treatment for breast cancer. Early detection and diagnosis and timely treatment are of great significance to improve the survival rate of breast cancer patients.

The aetiology of breast cancer is still not clear, and there are many related factors, such as individual differences and a lack of effective treatments. With the development of biomedicine, personalized medicine is becoming the direction of breast cancer treatment in the future. At present, the medical plan can only be formulated through the study of single gene expression and mutation information. However, this information cannot fully reflect the personalized interaction and regulation among genes. Because onset and progression of cancer are often caused by the disruption of important biological networks such as cell cycle and apoptosis, but not a single gene. Indeed, there is a new and cutting-edge field of medical research, called network medicine, whose basic idea is that human diseases are rarely caused by single molecular determinant, but more likely influenced by a network of interacting molecular determinants with the propensity to cluster 
together in the human interactome [7-9]. Gene-gene interaction networks can reveal the interaction relations and regulatory mechanisms among genes. And they have the irreplaceable function of the single-gene monitoring of information (such as expression and mutation) in many aspects [10]. Therefore, the mechanism of the occurrence and development of breast cancer can be explored through changes in the interactions between genes. In this paper, we constructed sample-specific networks of breast cancer samples by calculating the correlation coefficient of protein-coding gene pairs to explore the gene-gene interaction networks related to breast cancer stages and subtypes (see Fig.1).

The survival time of different patients with breast cancer is significantly different. At present, the 5-year survival rate of breast cancer patients in China has reached 83.2\%. However, the 5-year survival rate of advanced cancer patients and Basal-like cancer patients are significantly lower, so it is necessary to study the biomarkers that affect the prognosis of breast cancer. In 2009, Joel S. Parker et al. established a single-gene level survival analysis model to improve the prognosis of breast cancer and predict the efficacy of chemotherapy [11]. However, the robustness of the genebased model is not very high. Thus, this paper aims to establish a more stable prognostic analysis model of breast cancer patients through gene-gene interactions. We used the differential correlation coefficients to model the prognosis of breast cancer. Lasso regression is suitable for data analysis and model construction with many independent variables but a limited sample size [12]. In this study, we used a Lasso regression model to effectively reduce the dimensionality of large gene pairs and then identified the gene interactions related to the prognosis of breast cancer. Finally, a multivariate Cox proportional hazards regression analysis based on the gene interactions was carried out to predict the survival of patients with breast cancer (see Fig.1B). A prognosis model was established and it also performed very well on an independent validation data.

\section{Materials \& Methods}

\section{Datasets}

In this paper, the RNA sequencing (RNA-seq) data of 290 normal breast tissues was downloaded from the GTEx database (https://gtexportal.org/home/), and the RNA-seq data of 1093 breast cancer samples was downloaded from the TCGA database (https://portal.gdc.cancer.gov/). A human protein-protein interaction network was from the STRING database version 11.0 (https://string-db.org/), and gene sets of all available186 KEGG pathways were downloaded from the GSEA/MSigDB database (http: //software.broadinstitute.org/gsea/msigdb). In addition, the clinical information of the breast cancer patients was downloaded from the TCGA database, including TNM stage, prognosis survival time and other information. The 290 normal breast tissues were used as reference samples. The gene expression data sets of normal and cancer samples were both converted to the TPM form and contain 18006 genes in total. The independent validation data of the prognosis model was from the GSE3494 set in GEO Datasets, which contains 251 expression profiles of breast tumors by array. 


\section{Construction of sample-specific networks}

In this study, gene-gene interactions with high confidence (comprehensive score $>0.9$ ) were selected from the STRING database, which includes regulatory, physical and co-expression protein-protein interaction networks. Furthermore, the above gene-gene interactions with both genes in one of the 186 KEGG pathways were used as the background network (or template network), which contained 3257 genes in total. The sample-specific network method aimed to calculate the difference of the gene co-expression when the single cancer sample was added to a bunch of normal samples. In short, the sample-specific networks to be constructed are actually networks with significant perturbation edges of gene co-expression.

In the following analysis, sample-specific networks for breast cancer samples were constructed based on gene expression profiles by using the method introduced in reference [10] (see Fig.1A). First, using the gene expression data of $n$ reference samples, namely all the normal breast tissues data, the reference network can be constructed by calculating the correlation coefficient $P C C_{n}$ (the Pearson correlation coefficient (PCC)) of the gene pairs connected in the background network. The weights of the edges in the reference network are the PCC of the corresponding gene pairs. Then, the expression data of a single breast cancer sample was added to the reference samples, and the perturbed network of the single sample was constructed by calculating the new correlation coefficient $P C C_{n+1}$ of the gene pairs in the background network. For the single breast cancer sample, the differential correlation coefficients of each edge between the perturbed network and the reference network were calculated as: $\triangle P C C_{n}=P C C_{n+1}-P C C_{n}$, which called differential network for the sample. In reference [10], Liu et al. have proved that $\triangle P C C_{n}$ follows a normal distribution with a mean value of 0 and a variance of $\frac{1-P C C_{n}^{2}}{n-1}$ when $n$ is large enough. The significance level of each $\triangle P C C$ was determined by the $Z$-test. The statistical $Z$ value is calculated as follows with the null hypothesis that $\triangle P C C_{n}$ is equal to 0 :

$$
Z=\frac{\Delta P C C_{n}}{\left(1-P C C_{n}^{2}\right) /(n-1)} .
$$

Then, we can obtain the $P$-value for each gene pair from the $Z$-value. Gene pairs (or edges) were considered statistically significant if their $P$-values $<0.01$. All significant edges constitute the sample-specific network. Thus, adding the expression data of 1093 breast cancer samples to the reference samples one at a time, we finally constructed 1093 sample-specific networks.

\section{Identification of stage/subtype-related gene-gene interaction networks}

Only the gene pairs that are perturbed significantly in the most breast cancer samples are considered to be related to breast cancer. Then, the edges that are perturbed significantly in more than $90 \%$ of the samples by the binomial right-sided test $(P$-value $<0.05)$ constitute a gene-gene interaction network related to breast cancer. Specifically, we firstly divided the above breast cancer samples into different stages or subtypes based on TNM staging and PAM50 subtype system, secondly selected the perturbed significantly edges in more than $90 \%$ samples of different stages or subtypes, and then the stage/subtype gene-gene interaction networks were constructed. 
145

146

147

148

149

150

151

152

153

154

155

156

157

158

159

160

161

162

163

164

165

166

167

168

169

170

171

172

173

174

175

176

177

178

179

180

181

A slight change in the expression of high-degree genes in the network may cause disturbances of the entire network. Thus, these genes with high degree are considered to be the key genes for the onset and development of breast cancer. We selected genes with degrees $>5$ in the identified breast cancer-related network for the subsequent enrichment analysis. Furthermore, we also identified the key genes related to each TNM stage and PAM50 subtype with the same method. Here, because of the small number of stage V samples, stage V was combined with stage IV.

\section{Pathway enrichment analysis}

For the pathway enrichment analysis, we used the hypergeometric test as follows:

$$
p(m, M, N, n)=1-\sum_{i=0}^{m-1} \frac{\left(\begin{array}{c}
M \\
i
\end{array}\right)\left(\begin{array}{c}
N-M \\
n-i
\end{array}\right)}{\left(\begin{array}{l}
N \\
n
\end{array}\right)}
$$

where $N$ is the total number of genes in the background network, $M$ represents the number of key genes related to breast cancer (or a stage or subtype of breast cancer), $n$ accounts for the number of genes in a pathway, and $m$ represents the number of genes that both in the pathway and in key genes related to breast cancer (or a stage or subtype of breast cancer). Then, the pathway with $P$ value $<0.05$ was considered as significantly enriched in the breast cancer (or a stage or subtype of breast cancer) samples. Otherwise, we regarded that the pathway is not enriched in the corresponding group.

\section{Survival analysis by the Cox regression model}

Different from the usual survival analysis based on gene expression, the perturbation of gene co-expression $\triangle P C C$ (i.e. gene pairs or edges) was used to survival analysis. According to the clinical data of patients with breast cancer, we utilized the "survival" package and "survminer" package in R/Bioconductor to establish a univariate Cox proportional hazards regression model by setting patients' survival conditions (survival time and survival status) as the dependent variables and the $\triangle P C C$ of gene pairs in the differential network for each breast cancer samples as the covariates. Gene pairs with $P$-values $<0.05$ were considered to be related to the prognosis of breast cancer [13].

A large number of covariates may cause overfitting in establishing a multivariate Cox proportional hazards regression model; thus, using the least absolute shrinkage and selection operator (LASSO), we further selected the key gene pairs from these significant ones obtained by the univariate Cox proportional hazards analysis. LASSO is a common method used in highdimensional data regression, which can select prognosis-related gene pairs of breast cancer by shrinking regression coefficients. The tuning parameter $(\lambda)$ with the smallest mean-square error was selected by four-fold cross-validation to establish an optimal LASSO regression model. Then, the coefficients of most gene pairs reduced to zero, and a smaller number of gene pairs with nonzero coefficients were considered to be closely correlated with the prognosis of breast cancer.

LASSO Cox analysis was performed by using the "glmnet" package in R. Then, the risk score for each sample was calculated by the LASSO Cox regression model. According to the median risk score, breast cancer patients were divided into two groups (a high-risk group and a low-risk 
182

183

184

185

186

187

188

189

190

191

192

193

194

195

196

197

198

199

200

201

202

203

204

205

206

207

208

209

210

211

212

213

214

215

216

217

group). In addition, 234 breast tumors with relapse free survival information in the validation data set were analyzed by using the above sample-specific network method, and risk scores were calculated by the Cox regression model based on 1093 samples in TCGA. Then the validation samples were also divided into two groups in the same way. Finally, the corresponding KaplanMeier survival curves were plotted by using the packages "survminer" and "survival" in R.

\section{Results}

\section{Breast cancer-related gene-gene interaction networks}

The background network consisted of 46916 edges and 3237 genes. In addition, 2190 gene pairs were identified as significantly related to breast cancer, which constituted the gene-gene interaction network related to breast cancer (including 915 genes in total). We use the Cytoscape software to visualize the breast cancer-related network (see Fig.2).

Genes with degrees $>5$ in the breast cancer-related gene-gene interaction network (198 in total which are shown in Table S1). Among them, some genes with higher degrees $(>20)$ have been shown to be related to breast cancer. For example, CCNB1 has strong power to predict the survival of breast cancer patients with the phenotype of ER positive [1414]. The overexpression of GRB2 has been demonstrated to be significantly associated with the occurrence and poor prognosis of breast cancer [15]. PCNA has been proven to be a marker of proliferation in the diagnosis of breast cancer [16], SF3B4 has been shown to be a tumour suppressor, and somatic inactivating mutations occasionally occur in breast cancer [1717]. UBE2C may promote the development of breast cancer [18]. High Cdc20 and securin immune expression are associated with extremely poor outcomes in breast cancer patients [19], and overexpression of RPL17 affects breast cancer-associated brain metastases [20]. MAD2L1 may have great effect on breast cancer progression, and its expression might help to predicting breast cancer prognosis [2121]. The high expression of TRA2B is closely related to the cancer cell survival and therapeutic sensitivity of breast cancer [22]. GTF2H4 has been identified to be related to the survival risk of breast cancer [23].

\section{Stage-related gene-gene interaction networks}

The results of the four stage-related gene-gene interaction networks are shown in Fig.S1A-D. And the top 10 genes with the highest degrees in these four networks are displayed in Fig.S1E-H. There are obvious similarities and differences among the four stage-related gene interaction networks. There are 81 key genes shared by all stages (see Table S3), among which RPL17, CCNB1, and SF3B4 are genes that are highly (with degrees $>25$ ) related to breast cancer. Stage I has 5 specific genes: PSMC5, SDHB, RPL11, SDHA, and RPL13. Stage II has 3 specific genes: STX6, CCNA2, and CDC25C. Stage III has 4 specific genes: NDUFA6, EPN1, SF3A3, and LSM7. Stage IV has the largest number of specific genes, with a total of 38, among which CDC42, 
218 LSM2, NDUFS6, and CDC25A are strongly associated with it. And these stage-specific key genes 219 are shown in Table S4.

220 Subtype-related gene-gene interaction networks

221

222

223

224

225

226

227

228

229

230

231

232

233

234

235

236

237

238

239

240

241

242

243

244

245

246

247

248

249

250

251

252

253

254

255

256

The results of the four subtype-related gene interaction networks are shown in Fig.S2A-D. And the top 10 genes with the highest degrees in these four networks are displayed in Fig.S2E-H. The four subtype-related networks share similar and different characteristics. There are 34 key genes shared by the four subtypes (see Table S7). Among them, RPL17 and CCNB1 have higher degrees. The Luminal A subtype has 11 specific genes, including RPL23A, RPL10, and PRPF6, which are greatly related to it with higher degrees. The Luminal B subtype has 17 specific genes, including COX6C, EGFR, and CLTC, which are related to it with higher degrees. The Her2 subtype has 3 specific genes, NDUFA6, CCR8, and CASP3. The Basal-like subtype has the largest number of specific genes, 17 in total, including LSM2, DDX5, SF3A3, and MAGOH, with higher degrees. And these subtype-specific key genes are shown in Table S8.

\section{Pathways enriched in breast cancer patients}

There were 41 pathways (see Table S2) enriched in the breast cancer samples according to the pathway enrichment analysis, including some immune-related pathways, such as the Toll-like receptor signaling pathway, antigen processing and presentation, complement and coagulation cascades, the RIG-I-like receptor signaling pathway, and the cytosolic DNA-sensing pathway. Some important signal transduction and signal molecular interaction pathways were also included, such as the MAPK signaling pathway, Wnt signaling pathway, cytokine-cytokine receptor interaction, and ECM-receptor interaction pathways. Breast cancer is closely related to endocrine disorders [24], two endocrine-related pathways, adipocytokine signaling pathway, and PPAR signaling pathway, have also been identified as being related to breast cancer. In addition, some metabolic pathways, especially lipid metabolism pathways, have also been identified as being associated with breast cancer [25], such as the steroid hormone biosynthesis, arachidonic acid metabolism, arginine and proline metabolism pathway, and glycerolipid metabolism. Additionally, pathways in cancer was also enriched. The enrichment results are shown in Fig.3A.

Most of these pathways have been documented to be related to breast cancer. For example, the dysregulation of the steroid hormone biosynthesis pathway may affect steroid hormone levels and may thus be related to the susceptibility to breast cancer [24]. The PPAR signaling pathway may play an important role in the neoadjuvant chemotherapy response of breast cancer [26]. Mounting preclinical evidence supports targeting the MAPK signaling pathway in the triple negative breast cancer (TNBC) [27]. AMPK activators inhibit breast cancer cell proliferation by inhibiting DVL3-promoted Wnt/ $\beta$-catenin signaling pathway activity [28]. Toll-like receptors may play dual roles in human cancers [29]. The co-activation of the Hedgehog and Wnt signaling pathways is a poor prognostic marker in TNBC [30]. Prl-3 is closely related to cell migration and invasion in TNBC [31]. The YHD inhibition of 4T1 breast tumour growth may be related to the negative regulation of the JAK/STAT3 pathway by repressing the expression of IL- 6 and TGF- $\beta$ [32]. 
257

258

259

260

261

262

263

264

265

266

267

268

269

270

271

272

273

274

275

276

277

278

279

280

281

282

283

284

285

286

287

288

289

290

291

292

293

294

295

296

297

\section{Stage-related pathways}

The overlapping of pathways enriched in the four TNM stages are shown in Fig.3B. The proportion of enriched pathways shared by the four stages (see Table S5) is relatively high, including the Wnt signaling pathway, MAPK signaling pathway27, regulation of actin cytoskeleton, calcium signaling pathway34, pathways in cancer, and cell adhesion molecules, which have been shown to have a high correlation with breast cancer [27, 28, 33-35]. The pathways enriched in different stages are slightly different, especially Stage IV of breast cancer, which has 18 specific enriched pathways, among which the PPAR signaling pathway26, ECM-receptor interaction, tight junction, TGF-beta signaling pathway, NOD-like receptor signaling pathway, and other signaling pathways are mostly related to the metastases of breast cancer [26, 36-39].

As we expected, stage IV was specifically enriched the most pathways (18 in total, see Table S6) different from other stages. This result is probably because Stage IV breast cancer patients are the most serious, and their cancer cells are likely to have deteriorated and metastasized. Therefore, the disruption of the biological system balance of breast cancer patients at this stage is larger than that of other stages. Thus, the specific enriched pathways of Stage IV are correspondingly more.

\section{Subtype-related pathways}

The overlap and difference of the enriched pathways in the four PAM50 subtypes are shown in Fig.3C. There are slight differences in the subtype-related pathways. There are 4 enriched pathways shared by the four subtypes (see Table S9) including the cytokine-cytokine receptor interaction. As a special subtype of breast cancer, the Basal-like subtype (or TNBC) is characterized by high histological differentiation, a high risk of metastasis, a high recurrence rate, and a low survival rate. Probably due to the higher risk of Basal-like subtype, there are 9 specific pathways enriched in it, including the leukocyte transendothelial migration and chemokine signaling pathway. The subtype-specific enriched pathways are shown in Table S10.

\section{Prognosis-related gene pairs}

A total of 5652 gene pairs significantly related to the survival and prognosis of breast cancer were found by the univariate Cox proportional hazards model. In addition, 272 gene pairs were further identified by Lasso regression (see Fig.S3). A multivariate Cox proportional hazards regression model with these gene pairs as independent variables was constructed as follows. Score $=206.3 *($ ENO, PGK2 $)+35.9 *(E N 0$, PKLR $)+4.1 *(E B P$, HSD17B7 $)+5.5 *($ CYP1B, HSD17B1) $-3.4 *($ NDUFB2, NDUFB4) $-0.6 *($ ATP6V1A, ATP6V1B1 $)+\ldots$

The risk scores of the 1093 breast cancer patients in TCGA were calculated by this model. The median of the risk scores divided all patients into two groups. The corresponding KaplanMeier survival curve is shown in Fig.4A. Of note, survival analysis indicates that overall survival probability of patients with high risk scores is significantly lower than that with low risk scores $(P$-value $<0.0001)$.

In addition, the risk scores of the 234 breast tumors in the validation data set were also calculated by the above model with 264 gene pairs ( 8 gene pairs were omitted since these genes were not included in the expression profile of the validation data). In the same way, there are two groups with different scores. The relapse free survival probabilities of the two groups are significantly different $(P$-value $<0.0001)$, and the relapse free survival status of tumors in the low 
298

299

300

301

302

303

304

305

306

307

308

309

310

311

312

313

314

315

316

317

318

319

320

321

322

323

324

325

326

327

328

329

330

331

332

333

334

335

score group are all "alive" (see Fig. 4B). This result indicates that the prognosis model based on gene pairs can well predict the survival time of breast tumors in the independent validation data set.

\section{Discussion}

At present, research on cancer pathology is limited to gene expression and mutation information. However, the model of one gene to one disease is no longer suitable for the study of complex diseases. In fact, genes do not exist in isolation but participate in some complex biological networks, such as gene-gene interaction networks. Gene mutations or surroundings changes often affect the balance of gene interaction networks and the perturbation of the networks then affect the onset and development of complex diseases. Studies have shown that some genetic elements of breast cancer are related to nearby gene expression, such as some repetitive DNA in ER+/HER2breast cancer and transposable elements [40,41]. Therefore, network analysis can provide a more comprehensive and systematic point of view, to better understand the human disease onset and development mechanism.

Based on personalized medicine, Precision Medicine is a new medical concept and medical model, which needs to grasp the specific characteristic of different cancer samples accurately. The analysis of the biological network disturbance for each cancer patient conforms to the concept of precision medicine. In addition, the personalized medical treatment of breast cancer is in a relatively slow development stage.

In this paper, sample-specific networks of breast cancer samples were established to explore the gene-gene interaction networks related to the TNM stages and PAM50 subtypes of breast cancer. Then, the pathways related to breast cancer were identified by hypergeometric test. Through the same method, we also obtained the stage-related pathways and subtype-related pathways. Finally, the edge biomarkers (gene pairs) that are closely related to the prognosis of breast cancer were determined by using the LASSO regression model, and then a more stable prognostic analysis model was established by using these biomarkers. Our results indicate that the prognosis model has the robust and strong generalization capability, and it can be used in different gene expression data sets.

Many studies have shown that network-based methods are more robust and effective than single-gene-based methods, such as SWIM and WGCNA [42, 43]. SWIM is a tool able to extract from complex correlation networks the so-called "switch genes" that could be associated to the transition from physiological to a pathological condition. The WGCNA method plans to exploit the correlation patterns among genes. The advantages of network-based methods have been well documented and accepted in the analysis of noisy high-throughput data. Different from the usual network-based method, we made better use of a prior background network to explore the samplespecific networks. And the sample-specific networks are actually networks with significant perturbations edges of gene co-expression in our study, which is really very different from 
336 WGCNA. This study helps us to better understand the heterogeneity and mechanism of breast 337 cancer from an individual-level perspective. Precision medicine advocates the development of 338 individualized treatment according to the unique features of patients. Therefore, identifying the 339 unique pathogeny embedded in each patient is important to develop a treatment strategy for each 340 patient. Our sample-specific network analysis of breast cancer will promote the development of 341 precision medicine.

\section{Conclusions}

344

345

346

347

348

349

350

351

352

353

354

355

356

357

358

359

360

361

362 363

364

365

366

367

368

In this paper, the sample-specific network of each breast cancer sample was constructed based on network analysis, and further breast cancer (subtype/stage)-related gene-gene interaction networks were identified. The edge biomarkers (gene pairs) related to the prognosis of breast cancer were also identified and a risk prediction model was established based on these edge biomarkers finally.

This study develops an individualized network analysis for each patient which would promote a new train of thought and method for the precision medicine. This whole process of sample-specific network analysis using co-expression can also be used to analyze other cancers. However, the co-expression perturbation which used to construct sample-specific network, does not roundly measure the changes of gene interactions. So, we will consider further designing a method which can characterize the perturbation of gene interactions comprehensively. In addition, how to obtain subtype-specific networks (or stage-specific networks) from samplespecific networks based on network structure is still a problem worth considering.

\section{Acknowledgements}

We are very grateful to Prof. Zhaohui Qin for his useful advice.

\section{Additional information and declarations}

Funding: This work was supported by the China Postdoctoral Science Foundation (No. 2019M651658), and the National College Students' Innovation Training Program (No. 201910307068Z).

Author contributions: All authors discussed the results and commented on the manuscript. Conflicts of Interest: The authors declare no conflicts of interest.

Supplementary Materials: Supplementary information for this article are available in the supplemental files. Table S1: Key genes of BRCA (the genes with degrees $>5$ in the breast cancer- 
369

370

371

372

373

374

375

376

377

378

379

380

381

382

383

384

385

386

387

388

389

390

391

392

393

394

395

396

397

398

399

400

401

402

403

404

405

related gene-gene interaction network). Table S2: Pathways enriched in BRCA (the pathways with $P$-value $<0.05$ by the enrichment analysis in all breast cancer samples). Table S3: Key genes shared by four stages. Table S4: Stage-specific key genes. Table S5: Enriched pathways shared by four stages. Table S6: Stage-specific enriched pathways. Table S7: Key genes shared by four subtypes. Table S8: Subtype-specific key genes. Table S9: Enriched pathways shared by four subtypes. Table S10: Subtype-specific enriched pathways. Figure S1: Gene-gene interaction networks and bar charts of gene degrees related to breast cancer TNM stages. Figure S2: Genegene interaction networks and bar charts of gene degrees related to breast cancer PAM50 subtypes. Figure S3: Establishment of the LASSO regression model.

\section{References}

1. Bray F, Ferlay J, Soerjomataram I, Siegel RL, Torre LA, Jemal A. Global cancer statistics 2018: GL OBOCAN estimates of incidence and mortality worldwide for 36 cancers in 185 countries. CA Cancer J Clin, 2018. 68(6):394- 424.

2. Perou CM, Sorlie T, Eisen MB, van de Rijn M, Jeffrey SS, Rees CA, Pollack JR, Ross DT, Johnsen H, Akslen LA, Fluge O, Pergamenschikov A, Williams C, Zhu SX, Lønning PE, Børresen-Dale AL, Brown PO, Botstein D. Molecular portraits of human breast tumors. Nature, 2000. 406(6797): 747-752.

3. Hammond ME, Hayes DF, Dowsett M, Allred DC, Hagerty KL, Badve S, Fitzgibbons PL, Francis G, Goldstein NS, Hayes M, Hicks DG, Lester S, Love R, Mangu PB, McShane L, Miller K, Osborne CK, Paik S, Perlmutter J, Rhodes A, Sasano H, Schwartz JN, Sweep FC, Taube S, Torlakovic EE, Valenstein P, Viale G, Visscher D, Wheeler T, Williams RB, Wittliff JL, Wolff AC. American Society of Clinical Oncology/College of American Pathologists guideline recommendations for immunohistochemical testing of estrogen and progesterone receptors in breast cancer. J Clin Oncol, 2010.28(16):2784-95.

4. Fitzgibbons PL, Murphy DA, Hammond ME, Allred DC, Valenstein PN. Recommendations for validating estrogen and progesterone receptor immunohistochemistry assays. Arch Pathol Lab Med, 2010. 134(6):930-5.

5. Cheang MC, Chia SK, Voduc D, Gao D, Leung S, Snider J, Watson M, Davies S, Bernard PS, Parker JS, Perou CM, Ellis MJ, Nielsen TO. Ki-67 index, HER2 status, and prognosis of patients with luminal B breast cancer. $J$ Natl Cancer Inst, 2009. 101(10):736-750.

6. Goldhirsch A, Wood WC, Coates AS, Gelber RD, Thürlimann B, Senn HJ; Panel members. Strategies for subtypes-dealing with the diversity of breast cancer: highlights of the St.Gallen International Expert Consensus on the Primary Therapy of Early Breast Cancer. Ann Oncol, 2011. 22(8):1736-1747.

7. Albert-László Barabási, Natali Gulbahce, Joseph Loscalzo. Network medicine: a networkbased approach to human disease. Nature Reviews Genetics. 2011. 12:56-68. 
406 8. Conte F, Fiscon G, Licursi V, Bizzarri D, D'Antò T, Farina L, Paci P. A paradigm shift in 407 medicine: A comprehensive review of network-based approaches. Biochim Biophys Acta

408

409

410

411

412

413

414

415

416

417

418

419

420

421

422

423

424

425

426

427

428

429

430

431

432

433

434

435

436

437

438

439

440

441

442

443

Gene Regul Mech. 2019. 194416.

9. Giulia Fiscon, Federica Conte, Lorenzo Farina, Paola Paci. Network-Based Approaches to Explore Complex Biological Systems towards Network Medicine. Genes. 2018. 9(9):437.

10. Liu X, Wang Y, Ji H, Aihara K, Chen L. Personalized characterization of diseases using sample-specific networks. Nucleic Acids Research, 2016. 44(22): e164.

11. Parker JS, Mullins M, Cheang MC, Leung S, Voduc D, Vickery T, Davies S, Fauron C, He X, Hu Z, Quackenbush JF, Stijleman IJ, Palazzo J, Marron JS, Nobel AB, Mardis E, Nielsen TO, Ellis MJ, Perou CM, Bernard PS. Supervised Risk Predictor of Breast Cancer Based on Intrinsic Subtypes. J Clin Oncol, 2009. 27(8): 1160-1167.

12. Zhang, Shangli \& Zhang, Lili \& Qiu, Kuanmin \& Lu, Ying \& Cai, Baigen. Variable Selection in Logistic Regression Model. Chinese Journal of Electronics. 2015. 24(4):813-817.

13. Cheng P. A prognostic 3-long noncoding RNA signature for patients with gastric cancer. $J$ Cell Biochem. 2018. 119(11):9261-9269.

14. Ding K, Li W, Zou Z, Zou X, Wang C. CCNB1 is a prognostic biomarker for ER+ breast cancer. Med Hypotheses. 2014. 83(3):359-64.

15. Zhang Y, Xu G, Liu G, Ye Y, Zhang C, Fan C, Wang H, Cai H, Xiao R, Huang Z, Luo Q. miR-411-5p inhibits proliferation and metastasis of breast cancer cell via targeting GRB2. Biochem Biophys Res Commun. 2016. 476(4):607-613.

16. Juríková M, Danihel L', Polák Š, Varga I. Ki67, PCNA, and MCM proteins: Markers of proliferation in the diagnosis of breast cancer. Acta Histochem. 2016. 118(5):544-52.

17. Denu RA, Burkard ME. Synchronous Bilateral Breast Cancer in a Patient With Nager Syndrome. Clin Breast Cancer. 2017. 17(3):151-153.

18. Mo CH, Gao L, Zhu XF, Wei KL, Zeng JJ, Chen G, Feng ZB. The clinicopathological significance of UBE2C in breast cancer: a study based on immunohistochemistry, microarray and RNA-sequencing data. Cancer Cell Int. 2017. 17:83.

19. Karra H, Repo H, Ahonen I, Löyttyniemi E, Pitkänen R, Lintunen M, Kuopio T, Söderström M, Kronqvist P. Cdc20 and securin overexpression predict short-term breast cancer survival. Br J Cancer. 2014. 110(12):2905-13.

20. Yuan F, Wang W, Cheng H. Co-expression network analysis of gene expression profiles of HER2 $^{+}$breast cancer-associated brain metastasis. Oncol Lett. 2018. 16(6):7008-7019.

21. Wang Z, Katsaros D, Shen Y, Fu Y, Canuto EM, Benedetto C, Lu L, Chu WM, Risch HA, Yu H. Biological and Clinical Significance of MAD2L1 and BUB1, Genes Frequently Appearing in Expression Signatures for Breast Cancer Prognosis. PLoS One. 2015. 10(8):e0136246.

22. Best A, Dagliesh C, Ehrmann I, Kheirollahi-Kouhestani M, Tyson-Capper A, Elliott DJ. Expression of Tra2 $\beta$ in Cancer Cells as a Potential Contributory Factor to Neoplasia and Metastasis. Int J Cell Biol. 2013. 2013:843781.

PeerJ reviewing PDF | (2020:03:46498:1:1:NEW 10 Apr 2020) 
444

445

446

447

448

449

450

451

452

453

454

455

456

457

458

459

460

461

462

463

464

465

466

467

468

469

470

471

472

473

474

475

476

477

478

479

480

481

23. Ge J, Liu H, Qian D, Wang X, Moorman PG, Luo S, Hwang S, Wei Q. Genetic variants of genes in the NER pathway associated with risk of breast cancer: A large-scale analysis of 14 published GWAS datasets in the DRIVE study. Int J Cancer. 2019. 145(5):1270-1279.

24. Sakoda LC, Blackston C, Doherty JA, Ray RM, Lin MG, Stalsberg H, Gao DL, Feng Z, Thomas DB, Chen C. Polymorphisms in steroid hormone biosynthesis genes and risk of breast cancer and fibrocystic breast conditions in Chinese women. Cancer Epidemiol Biomarkers Prev. 2008. 17(5):1066-73.

25. Merdad A, Karim S, Schulten HJ, Jayapal M, Dallol A, Buhmeida A, Al-Thubaity F, GariI MA, Chaudhary AG, Abuzenadah AM, Al-Qahtani MH. Transcriptomics profiling study of breast cancer from Kingdom of Saudi Arabia revealed altered expression of Adiponectin and Fatty Acid Binding Protein4: Is lipid metabolism associated with breast cancer? BMC Genomics. 2015. 16 Suppl 1: S11.

26. Chen YZ, Xue JY, Chen CM, Yang BL, Xu QH, Wu F, Liu F, Ye X, Meng X, Liu GY, Shen $\mathrm{ZZ}$, Shao ZM, Wu J. PPAR signaling pathway may be an important predictor of breast cancer response to neoadjuvant chemotherapy. Cancer Chemother Pharmacol. 2012. 70(5):637-44.

27. Giltnane JM, Balko JM. Rationale for targeting the Ras/MAPK pathway in triple-negative breast cancer. Discov Med. 2014. 17(95):275-83.

28. Zou YF, Xie CW, Yang SX, Xiong JP. AMPK activators suppress breast cancer cell growth by inhibiting DVL3-facilitated Wnt/ $\beta$-catenin signaling pathway activity. Mol Med Rep. 2017. 15(2):899-907.

29. Khademalhosseini M, Arababadi MK. Toll-like receptor 4 and breast cancer: an updated systematic review. Breast Cancer. 2019. 26(3):265-271.

30. Bhateja P, Cherian M, Majumder S, Ramaswamy B. The Hedgehog Signaling Pathway: A Viable Target in Breast Cancer? Cancers (Basel). 2019. 11(8):1126.

31. Gari HH, DeGala GD, Ray R, Lucia MS, Lambert JR. PRL-3 engages the focal adhesion pathway in triple-negative breast cancer cells to alter actin structure and substrate adhesion properties critical for cell migration and invasion. Cancer Lett. 2016. 380(2):505-12.

32. Mao D, Feng L, Gong H. The Antitumor and Immunomodulatory Effect of Yanghe Decoction in Breast Cancer Is Related to the Modulation of the JAK/STAT Signaling Pathway. Evid Based Complement Alternat Med. 2018. 2018:8460526.

33. Kazazian K, Go C, Wu H, Brashavitskaya O, Xu R, Dennis JW, Gingras AC, Swallow CJ. Plk4 Promotes Cancer Invasion and Metastasis through Arp2/3 Complex Regulation of the Actin Cytoskeleton. Cancer Res. 2017. 77(2):434-447.

34. Woltmann A, Chen B, Lascorz J, Johansson R, Eyfjörd JE, Hamann U, Manjer J, EnquistOlsson K, Henriksson R, Herms S, Hoffmann P, Hemminki K, Lenner P, Försti A. Systematic pathway enrichment analysis of a genome-wide association study on breast cancer survival reveals an influence of genes involved in cell adhesion and calcium signaling on the patients' clinical outcome. PLoS One. 2014. 9(6): e98229.

PeerJ reviewing PDF | (2020:03:46498:1:1:NEW 10 Apr 2020) 
482

483

484

485

486

487

488

489

490

491

492

493

494

495

496

497

498

499

500

501

502

503

504

505

506

35. Saadatmand S, de Kruijf EM, Sajet A, Dekker-Ensink NG, van Nes JG, Putter H, Smit VT, van de Velde CJ, Liefers GJ, Kuppen PJ. Expression of cell adhesion molecules and prognosis in breast cancer. Br J Surg. 2013. 100(2):252-60.

36. Bao Y, Wang L, Shi L, Y un F, Liu X, Chen Y, Chen C, Ren Y, Jia Y. Transcriptome profiling revealed multiple genes and ECM-receptor interaction pathways that may be associated with breast cancer. Cell Mol Biol Lett. 2019. 24:38.

37. Yang Y, Liu L, Fang M, Bai H, Xu Y. The chromatin remodeling protein BRM regulates the transcription of tight junction proteins: Implication in breast cancer metastasis. Biochim Biophys Acta Gene Regul Mech. 2019. 1862(5):547-556.

38. Tang X, Shi L, Xie N, Liu Z, Qian M, Meng F, Xu Q, Zhou M, Cao X, Zhu WG, Liu B. SIRT7 antagonizes TGF- $\beta$ signaling and inhibits breast cancer metastasis. Nat Commun. 2017. $8(1): 318$.

39. Peng L, Hu Y, Chen D, Linghu R, Wang Y, Kou X, Yang J, Jiao S. Ubiquitin specific protease 21 upregulation in breast cancer promotes cell tumorigenic capability and is associated with the NOD-like receptor signaling pathway. Oncol Lett. 2016. 12(6):4531-4537.

40. Yandım C, Karakülah G. Dysregulated expression of repetitive DNA in ER+/HER2- breast cancer. Cancer Genet. 2019. 239:36-45.

41. Karakülah G, Arslan N, Yandım C, Suner A. TEffectR: an R package for studying the potential effects of transposable elements on gene expression with linear regression model. PeerJ. 2019. 7: e8192.

42. Paci P, Colombo T, Fiscon G, Gurtner A, Pavesi G, Farina L. SWIM: a computational tool to unveiling crucial nodes in complex biological networks. Sci Rep. 2017. 7(1):44797.

43. Langfelder P, Horvath S. WGCNA: an R package for weighted correlation network analysis. BMC Bioinformatics. 2008. 9(1):559. 


\section{Figure Legends}

\section{Figure 1}

An integrative framework identifying breast cancer-related gene-gene interaction networks.

(A) Construction of sample-specific networks based on gene expression data. A reference network can be established based on the expression profiles of $n$ reference samples by calculating the correlation coefficients $P C C_{n}$ of gene pairs. Then, adding a new sample $s d_{x}$ into the reference samples, a perturbed network is established by calculating the new correlation $P C C_{n+1}$ of the $n+1$ samples. Because of sample $s d_{x}$, the perturbed network is different from the reference network, and the difference $\triangle P C C_{n}($ $\left.P C C_{n+1}-P C C_{n}\right)$ of each edge in the background network constitutes the differential network. Then, the significance of each edge can be quantified by a statistical Z-test. The sample-specific network for sample $s d_{x}$ is composed by those edges with significant $\Delta$ $P C C_{n}$.

(B) The framework to identify the breast cancer-related gene-gene interaction network based on gene expression. Using the sample-specific network analysis method, $m$ cancer sample-specific networks were constructed. Then, these constructed sample-specific networks were analysed to identify breast cancer-related networks, stage-related networks and subtype-related networks, as well as gene-interaction biomarkers associated with the prognosis of breast cancer. Moreover, pathway enrichment analysis based on KEGG pathways and survival analysis based on the LASSO regression model were performed.

\section{Figure 2}

Gene-gene interaction networks related to breast cancer. Nodes in these networks stand for genes, and the size of the nodes corresponds to the degree of the genes in the network. The purple nodes represent the genes with degrees $\geq 15$, and the blue ones are the genes with degrees $<15$. 


\section{Figure 3}

Pathways enriched in breast cancer, as well as different stages and subtypes of it.

(A) KEGG pathways enriched in breast cancer samples, ranked by -log10(p).

(B) Overlap and difference of the enriched pathways in the four breast cancer stages.

There are 11 commonly enriched pathways in the four stages. The number of Stage IVspecific pathways was 18.

(C) Overlap and difference of the enriched pathways in the four PAM50 subtypes. There are 4 commonly enriched pathways in the four PAM50 subtypes. The number of Basallike specific pathways is 9 .

\section{Figure 4}

Kaplan-Meier survival analysis.

(A) Kaplan-Meier survival plots for two different groups of breast cancer patients in TCGA. The $X$ axis is survival days. The $Y$ axis is overall survival rate.

(B) Kaplan-Meier survival plots for two different groups of breast tumors in the independent validation data set. The $X$ axis is relapse free survival time (days). The $Y$ axis is relapse free survival rate.

Figure S1. Gene-gene interaction networks related to breast cancer TNM stages. Nodes in these networks stand for genes, and the size of the nodes corresponds to the degree of the genes in the network. The purple nodes represent the genes with degrees $\geqslant 15$, and the blue ones are the genes with degrees $<15$.

(A-D) Gene-gene interaction networks associated with Stage I, II, III, and IV respectively. (E-H) The bar charts of top 10 genes with the highest degrees in gene-gene interaction networks related to Stage I, II, III, and IV. The $Y$ axis is gene, and the $X$ axis is the gene degree.

Figure S2. Gene-gene interaction networks related to breast cancer PAM50 subtypes. Nodes in these networks stand for genes, and the size of the nodes 
corresponds to the degree of the genes in the network. The purple nodes represent the genes with degrees $\geqslant 15$, and the blue ones are the genes with degrees $<15$.

(B-D) Gene-gene interaction networks associated with LumA, LumB, Her2, and Basallike subtypes respectively.

$(E-H)$ The bar charts of top 10 genes with the highest degrees in gene-gene interaction networks related to LumA, LumB, Her2, and Basal-like subtypes. The $Y$ axis is gene, and the $X$ axis is the gene degree.

Figure S3. Establishment of the LASSO regression model.

(A) Four-fold cross-validation for tuning parameter $(\lambda)$ selection in the LASSO model.

(B) LASSO coefficient profiles of 272 gene interactions. 


\section{Figure 1}

An integrative framework identifying breast cancer-related gene-gene interaction networks.

(A) Construction of sample-specific networks based on gene expression data. A reference network can be established based on the expression profiles of $n$ reference samples by calculating the correlation coefficients $P C C_{n}$ of gene pairs. Then, adding a new sample $s d_{x}$ into the reference samples, a perturbed network is established by calculating the new correlation $P C C_{n+1}$ of the $n+1$ samples. Because of sample $s d_{x}$, the perturbed network is different from the reference network, and the difference $\triangle P C C_{n}\left(P C C_{n+1}-P C C_{n}\right)$ of each edge in the background network constitutes the differential network. Then, the significance of each edge can be quantified by a statistical Z-test. The sample-specific network for sample sdx is composed by those edges with significant $\triangle P C C_{n^{*}}$ (B) The framework to identify the breast cancer-related gene-gene interaction network based on gene expression. Using the samplespecific network analysis method, $m$ cancer sample-specific networks were constructed. Then, these constructed sample-specific networks were analysed to identify breast cancerrelated networks, stage-related networks and subtype-related networks, as well as geneinteraction biomarkers associated with the prognosis of breast cancer. Moreover, pathway enrichment analysis based on KEGG pathways and survival analysis based on the LASSO regression model were performed. 


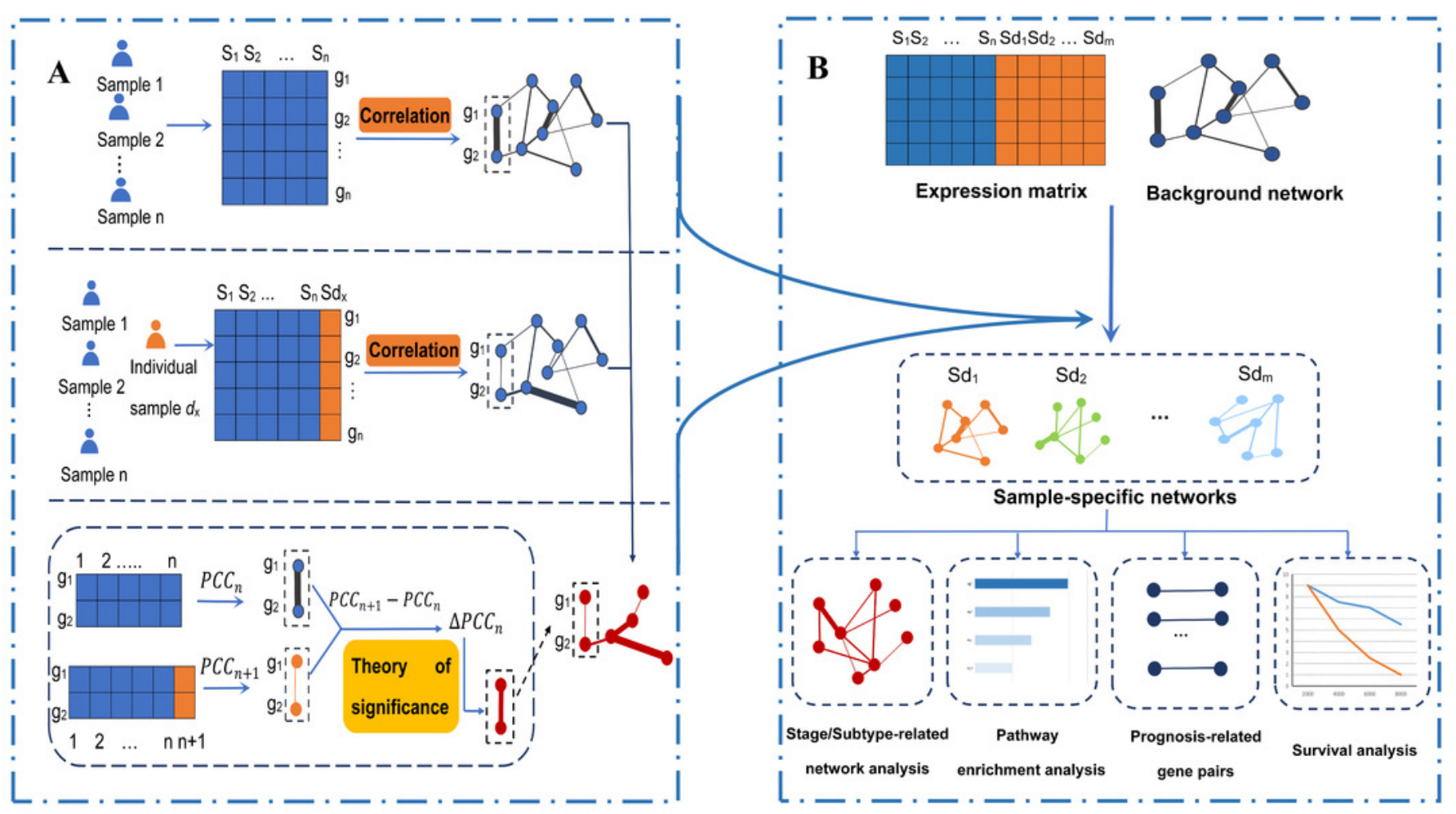


Figure 2

Gene-gene interaction networks related to breast cancer.

Nodes in these networks stand for genes, and the size of the nodes corresponds to the degree of the genes in the network. The purple nodes represent the genes with degrees $\geq$ 15 , and the blue ones are the genes with degrees $<15$.

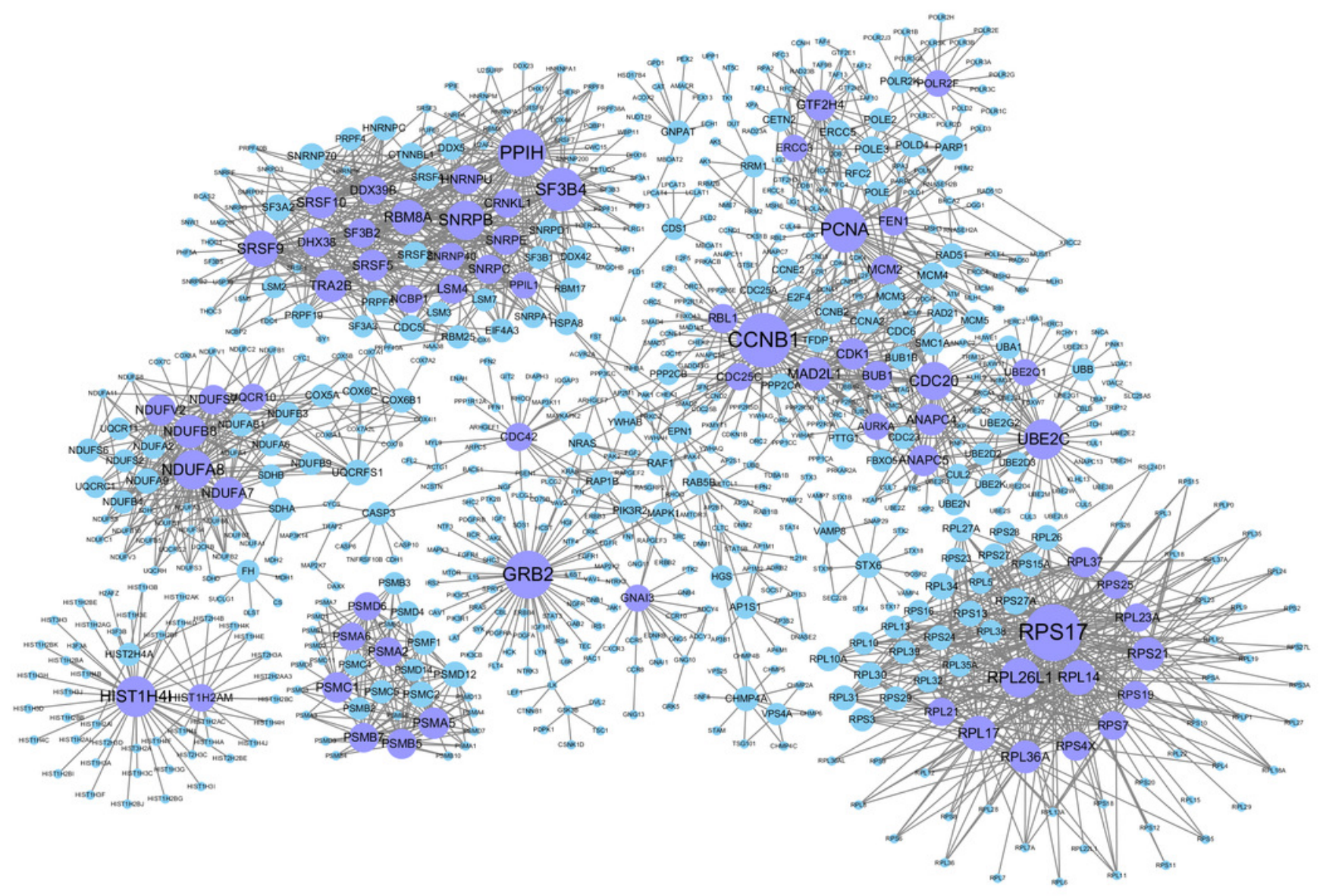




\section{Figure 3}

Pathways enriched in breast cancer, as well as different stages and subtypes of it.

(A) KEGG pathways enriched in breast cancer samples, ranked by -log10(p).(B) Overlap and difference of the enriched pathways in the four breast cancer stages. There are 11 commonly enriched pathways in the four stages. The number of Stage IV-specific pathways was 18.(C) Overlap and difference of the enriched pathways in the four PAM50 subtypes. There are 4 commonly enriched pathways in the four PAM50 subtypes. The number of Basal-like specific pathways is 9 . 
A

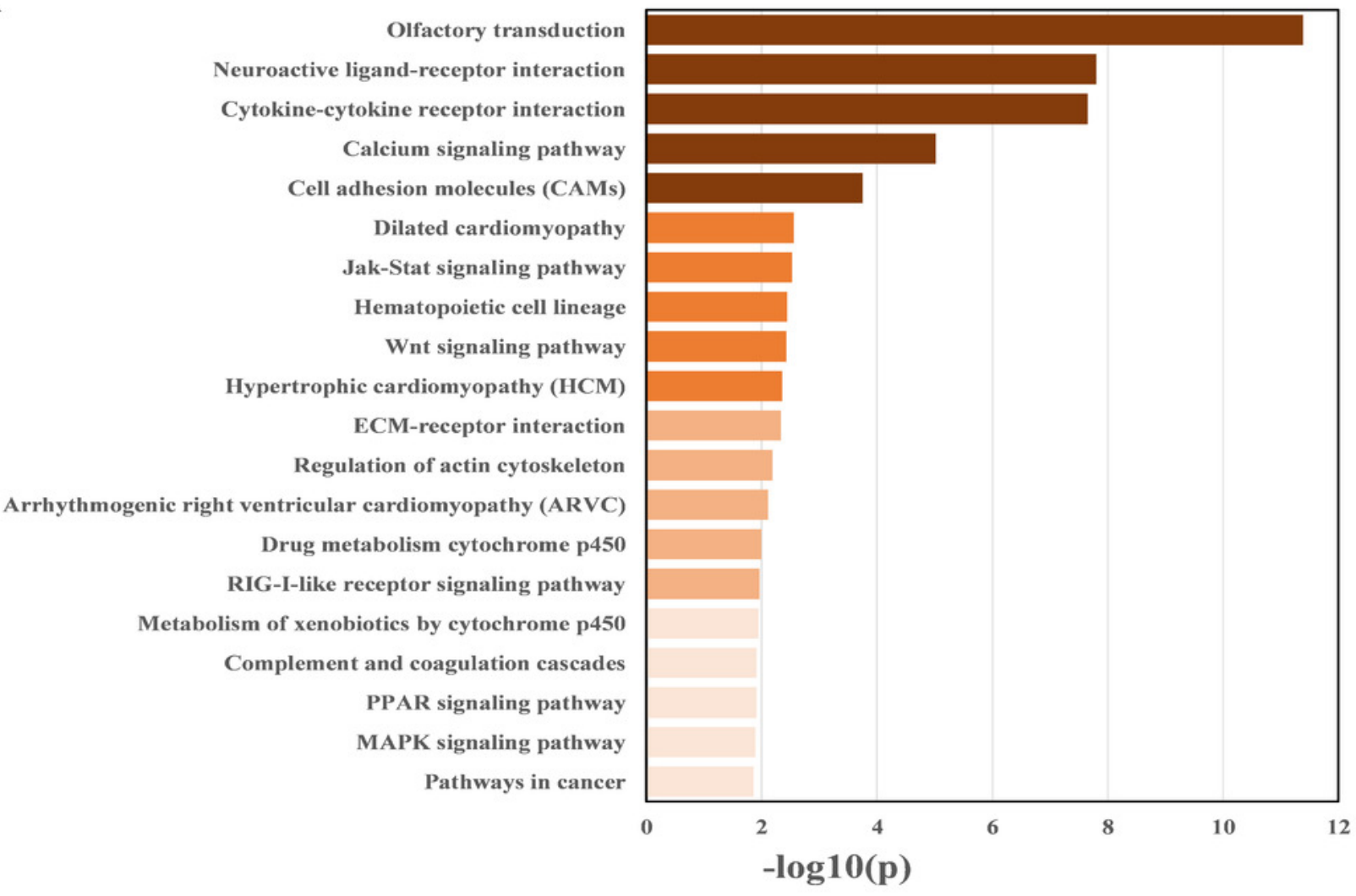

B

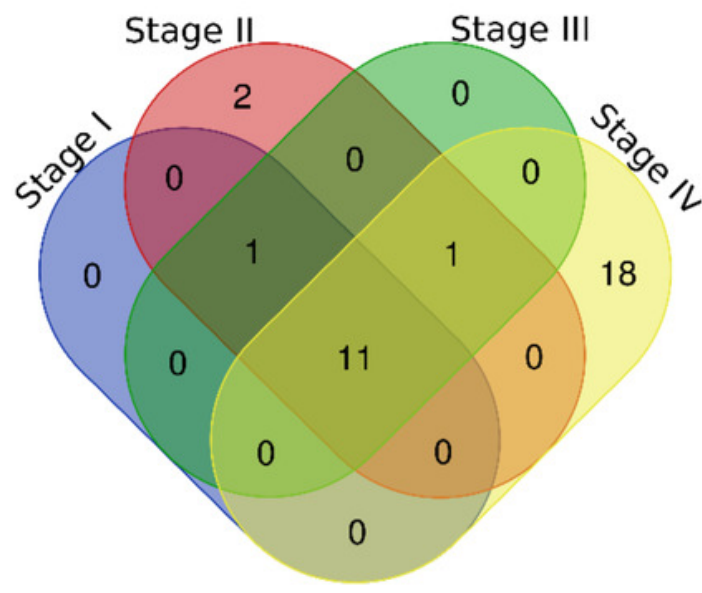

C

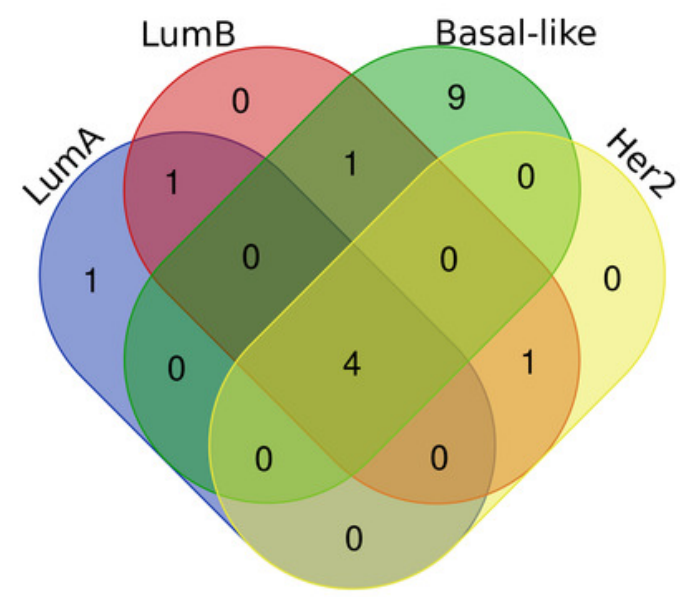




\section{Figure 4}

Kaplan-Meier survival analysis.

(A) Kaplan-Meier survival plots for two different groups of breast cancer patients in TCGA.The $X$ axis is survival days. The $Y$ axis is overall survival rate. (B) Kaplan-Meier survival plots for two different groups of breast tumors in the independent validation data set. The $X$ axis is relapse free survival time (days). The $Y$ axis is relapse free survival rate. 


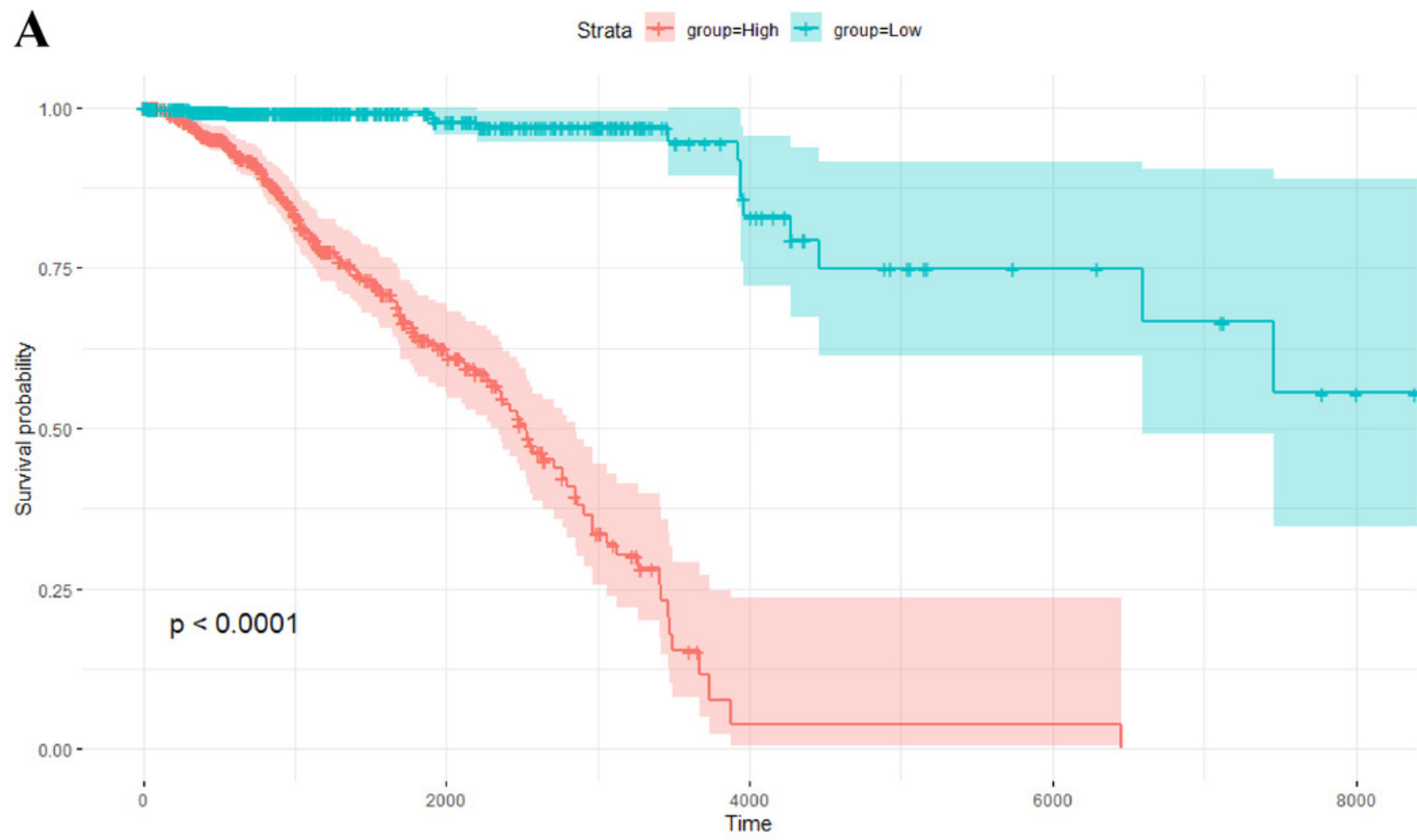

B

Strata + group=High + group=Low

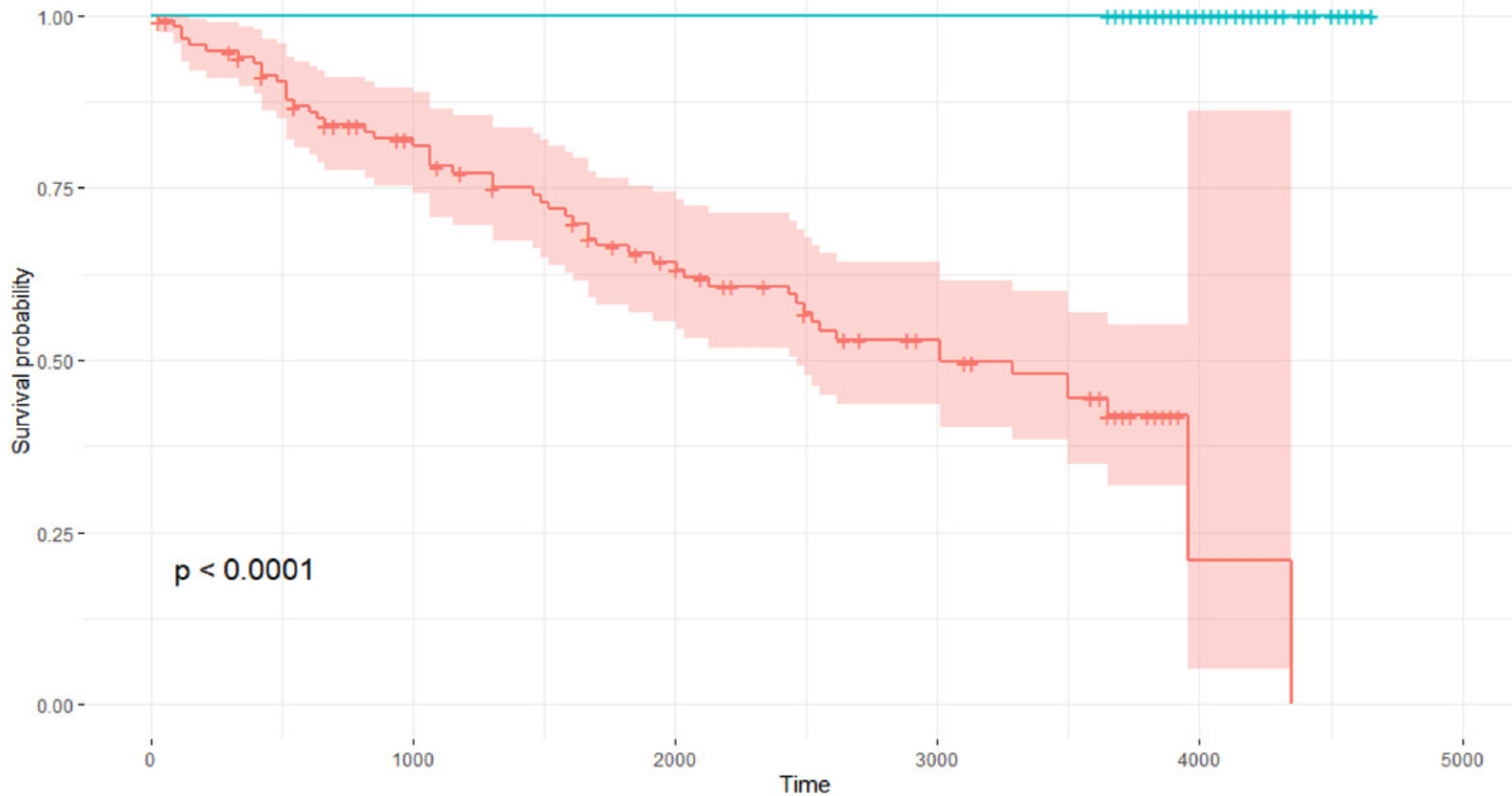

